\title{
A avaliação das políticas esportivas de alto rendimento: uma revisão de literatura
}

\author{
The evaluation of high performance sports policies: a literature review \\ La evaluación de las políticas deportivas de alto rendimiento: una revisión de la literatura
}

Recebido: 08/12/2021 | Revisado: 12/12/2021 | Aceito: 17/12/2021 | Publicado: 02/01/2022

\author{
Natasha Santos-Lise \\ ORCID: https://orcid.org/0000-0002-3352-0673 \\ Universidade Estadual de Ponta Grossa, Brasil \\ E-mail: natashaslise@gmail.com \\ Fernando Marinho Mezzadri \\ ORCID: https://orcid.org/0000-0001-8601-5227 \\ Universidade Federal do Paraná, Brasil \\ E-mail: fmezzadri@uol.com.br \\ Marco Antonio Ribas Cavalieiri \\ ORCID: https://orcid.org/0000-0002-4251-6879 \\ Universidade Federal do Paraná, Brasil \\ E-mail: cavaga@uol.com.br \\ Sérgio Wynnek Junior \\ ORCID: https://orcid.org/0000-0001-5784-1108 \\ Universidade Estadual de Ponta Grossa, Brasil \\ E-mail: swynnek92@gmail.com
}

\begin{abstract}
Resumo
O presente artigo busca apresentar uma revisão da literature, referente à avaliação das políticas esportivas, especificamente ligadas ao alto rendimento. Esta se refere a uma pesquisa maior que busca identificar o modelo lógico do Programa Bolsa-Atleta, a fim de pensar as possibilidades de avaliação de tal política. Por este motivo, o recorte da busca foi o esporte de alto rendimento. A busca foi realizada na base de dados Scopus, Google Acadêmico e sites das revistas científicas brasileiras. Os termos utilizados foram "process evaluation sports policy" e "evidence based evaluation sports policy". Respectivamente, foram encontrados 119 e 65 textos. Com base na leitura dos resumos, selecionou-se 25 artigos correspondentes a cada termo, totalizando um corpus de investigação de 50 artigos. Entre estes, permaneceram títulos que apontavam para a reflexão sobre a avaliação no esporte de inclusão e/ou participação, sem tratar especificamente o esporte de alto rendimento. Assim, foi realizada uma nova leitura dos resumos, o que reduziu ainda mais o referido corpus. Extraindo-se as repetições de textos que apareceram na busca de ambos os termos, foram seis artigos selecionados, para "evidence based evaluation sports policy", e nove, para "process evaluation sports policy" - o que totalizou 15 artigos, os quais foram lidos integralmente. Nesse sentido, é possível inferir uma escassa produção acerca da avaliação das políticas esportivas, em um sentido geral, e mais ainda em se tratando do esporte de alto rendimento.
\end{abstract}

Palavras-chave: Avaliação baseada em evidências; Avaliação de processo; Política pública; Esporte.

\begin{abstract}
This article seeks to present a review of the literature, referring to the evaluation of sports policies, specifically linked to high performance. This refers to a larger research that seeks to identify the logical model of the Bolsa-Atleta Program, in order to consider the possibilities for evaluating such a policy. For this reason, the search was focused on high performance sport. The search was carried out in the Scopus database, Academic Google and websites of Brazilian scientific journals. The terms used were "process evaluation sports policy" and "evidence based evaluation sports policy". Respectively, 119 and 65 texts were found. Based on the reading of the abstracts, 25 articles corresponding to each term were selected, totaling a research corpus of 50 articles. Among these, titles that pointed to the reflection on evaluation in the sport of inclusion and/or participation, without specifically dealing with highperformance sport, remained. Thus, the abstracts were read again, which further reduced the aforementioned corpus. Extracting the repetitions of texts that appeared in the search for both terms, six articles were selected, for "evidence based evaluation sports policy", and nine, for "process evaluation sports policy" - totaling 15 articles, which were read in full. In this sense, it is possible to infer that there is little production about the evaluation of sports policies, in a general sense, and even more in terms of high performance sport.
\end{abstract}

Keywords: Evidence based evaluation; Process evaluation; Public policy; Sport.

\section{Resumen}

Este artículo busca presentar una revisión de la literatura, referida a la evaluación de las políticas deportivas, específicamente vinculadas al alto rendimiento. Se trata de una investigación más amplia que busca identificar el 
modelo lógico del Programa Bolsa-Atleta, con el fin de considerar las posibilidades de evaluar dicha política. Por este motivo, la búsqueda se centró en el deporte de alto rendimiento. La búsqueda se realizó en la base de datos Scopus, Academic Google y sitios web de revistas científicas brasileñas. Los términos utilizados fueron "política deportiva de evaluación de procesos" y "política deportiva de evaluación basada en evidencias". Se encontraron, respectivamente, 119 y 65 textos. A partir de la lectura de los resúmenes se seleccionaron 25 artículos correspondientes a cada término, totalizando un corpus de investigación de 50 artículos. Entre estos, quedaron títulos que apuntaban a la reflexión sobre la evaluación en el deporte de inclusión y / o participación, sin abordar específicamente el deporte de alto rendimiento. Así, se volvieron a leer los resúmenes, lo que redujo aún más el corpus mencionado. Extrayendo las repeticiones de los textos que aparecieron en la búsqueda de ambos términos, se seleccionaron seis artículos, para "política deportiva de evaluación basada en evidencias", y nueve, para "política deportiva de evaluación de procesos", totalizando 15 artículos, los cuales fueron leídos en su totalidad. En este sentido, es posible inferir que hay poca producción sobre la evaluación de las políticas deportivas, en un sentido general, y más aún en cuanto al deporte de alto rendimiento.

Palabras clave: Evaluación basada en evidencia; Evaluación del proceso; Política pública; Deporte.

\section{Introdução}

A avaliação tem sido considerada por muitos estudos (Foley 1992; Weiss 1993; O’Brien, 2013; Chen, 2018) uma fase imprescindível no ciclo político (Frey, 2000), sobretudo, no momento de tomada de decisão, por possibilitar aos policy-makers uma perspectiva mais direcionada quanto ao impacto de determinada ação. Junto a tal aspecto, tem se revelado - já desde a década de 1990 - a emergência de políticas baseadas em evidências, as quais permitem que os policy-makers construam intervenções pertinentes a um público-alvo específico.

Segundo Shushu Chen (2018), baseado em Stufflebeam e Coryn (2014), até o ano de 2014, foram levantadas 23 abordagens sobre avaliação. O ponto crucial - que, inclusive, é o foco de Chen (2018) -, que gera discussão se refere à qual abordagem escolher para avaliar uma política ou programa esportivos.

Tendo em vista todo o debate acerca da relevância do processo de avaliação e monitoramento, surgiu o questionamento acerca de como são avaliadas as políticas de alto rendimento no Brasil. Ao centrar, especificamente, no Programa Bolsa-Atleta - hoje, a maior política esportiva brasileira -, percebeu-se a existência mais de uma prestação de contas - relacionada a número de bolsas, montante financeiro e quantidade de atletas beneficiados -; do que propriamente de uma avaliação acerca do impacto que essas bolsas podem gerar no meio esportivo do país.

Nesse sentido, buscou-se identificar, por meio de um levantamento de produções científicas, a partir de uma revisão sistemática (Gomes \& Caminha, 2014), o que há disponível no meio acadêmico, quanto à avaliação de programas do esporte de alto rendimento - para que, então, fosse possível apontar indicadores que auxiliassem na avaliação do referido programa.

\section{Metodologia}

Do ponto de vista metodológico, portanto, a revisão sistemática (Gomes \& Caminha, 2014) acerca da avaliação de programas esportivos consistiu em quatro etapas: primeiramente, buscou-se, a partir de palavras-chave específicas, na plataforma de busca Scopus, nos sites das revistas científicas brasileiras e no google acadêmico. Posteriormente, fez-se uma pré-seleção dos textos que se enquadravam no enfoque estabelecido - qual seja, a avaliação dos programas esportivos de alto rendimento -, a partir da leitura dos resumos. O próximo passo foi ler os textos completos selecionados. A quarta etapa se deu a partir da leitura dos artigos, majoritariamente em língua inglesa, indicando a necessidade de realizar uma nova busca, com outros termos.

Deste modo, após a seleção da base, os passos adotados são apresentados na Figura 1:

Figura 1 - Fluxograma dos passos realizados na coleta de dados das investigações. 


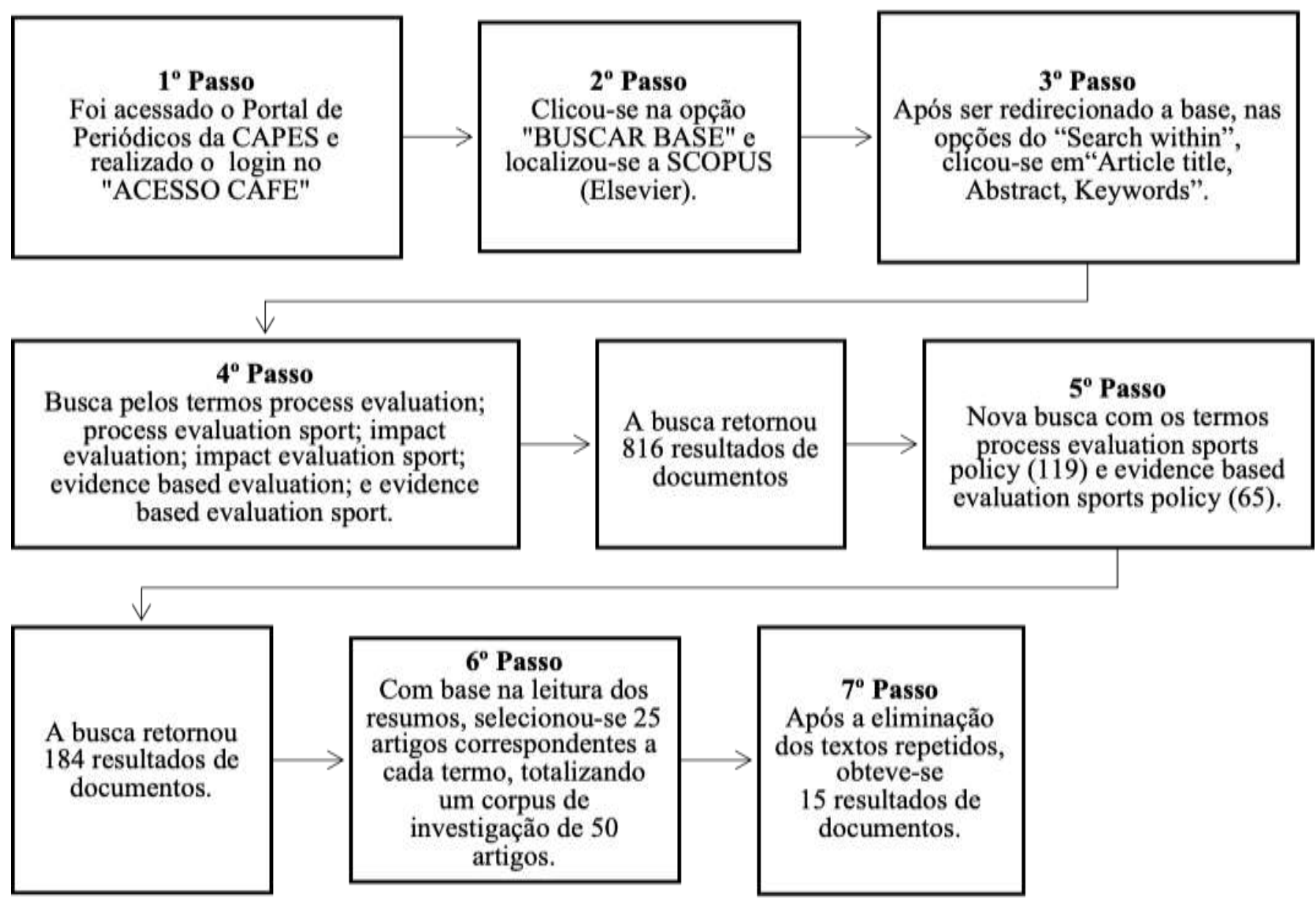

Fonte: Autores.

Os termos de busca utilizados na plataforma Scopus, foram delimitados com base no interesse inicial da pesquisa, em identificar estudos relacionados à avaliação de impacto, de processo e baseada em evidências. Assim, os termos de busca primeiramente selecionados foram: process evaluation (28 resultados); process evaluation sport (1 resultado); impact evaluation (5 resultados); impact evaluation sport (nenhum resultado); evidence based evaluation (5 resultados); e evidence based evaluation sport (777 resultados). O mecanismo de busca da referida plataforma englobou títulos, resumos e palavraschave definidas pelos autores, na submissão do texto, o que ampliou o alcance da procura pelos termos de busca selecionados.

Esses primeiros resultados encontrados apontaram para a necessidade de maior delimitação dos termos de busca, já que apareceram inúmeros artigos que não estavam relacionados ao esporte, e muitos destes apareceram repetidamente nas diferentes buscas. Dessa forma, optou-se pelos termos process evaluation sports policy e evidence based evaluation sports policy. O acréscimo da palavra "policy" permitiu que a procura se desse de forma mais direcionada, otimizando os resultados. Respectivamente, foram encontrados 119 e 65 textos. Com base na leitura dos resumos, selecionou-se 25 artigos correspondentes a cada termo, totalizando um corpus de investigação de 50 artigos. Entre estes, permaneceram títulos que 
apontavam para a reflexão sobre a avaliação no esporte de inclusão e/ou participação, sem tratar especificamente o esporte de alto rendimento. Assim, foi realizada uma nova leitura dos resumos, o que reduziu ainda mais o referido corpus. Extraindo-se as repetições de textos que apareceram na busca de ambos os termos, foram seis artigos selecionados, para evidence based evaluation sports policy, e nove, para process evaluation sports policy - o que totalizou 15 artigos, os quais foram lidos integralmente.

\section{Resultados e Discussão}

Conforme relatado na metodologia, após o levantamento e a seleção dos textos, foi possível desenvolver os Quadros 1,2 e 3, expostos a seguir. No Quadro 1, é possível verificar os seis artigos selecionados, de acordo com os critérios de inclusão estabelecidos, com relação ao termo "evidence based evaluation sports policy". 
Research, Society and Development, v. 11, n. 1, e5111124491, 2022

(CC BY 4.0) | ISSN 2525-3409 | DOI: http://dx.doi.org/10.33448/rsd-v11i1.24491

Quadro 1 - Artigos referentes ao termo de busca "evidence based evaluation sports policy".

\begin{tabular}{|c|c|c|c|c|c|}
\hline \multicolumn{6}{|c|}{ BASE DE DADOS SCIVERSE SCOPUS DA ELSEVIER - Evidence Based Evaluation Sports Policy } \\
\hline ARTIGO & $\operatorname{AUTOR}(\mathbf{E S})$ & ANO & PERIÓDICO & OBJETIVO & PROGRAMA/PAÍS \\
\hline $\begin{array}{l}\text { An evidence-based approach to physical activity } \\
\text { promotion and policy development in Europe: } \\
\text { contrasting case studies. }\end{array}$ & $\begin{array}{l}\text { CAVILL, N.; } \\
\text { FOSTER, C.; } \\
\text { OJA, P.; } \\
\text { MARTIN, B. W. }\end{array}$ & 2006 & $\begin{array}{l}\text { IUHPE - } \\
\text { Promotion \& } \\
\text { Education }\end{array}$ & $\begin{array}{l}\text { Caracterizar as quarto funções que } \\
\text { formam a base da abordagem sistemática } \\
\text { baseada em evidência, na promoção da } \\
\text { atividade física na Suiça, Finlândia e } \\
\text { Inglaterra. }\end{array}$ & Finlândia, Inglaterra e Suíça \\
\hline $\begin{array}{c}\text { 'Evidence. What evidence?': evidencebased policy } \\
\text { making and School Sport Partnerships in North West } \\
\text { England. }\end{array}$ & $\begin{array}{l}\text { SMITH, A.; } \\
\text { LEECH, R. }\end{array}$ & 2010 & $\begin{array}{c}\text { International } \\
\text { Journal of Sport } \\
\text { Policy and Politics }\end{array}$ & $\begin{array}{l}\text { School Sport Partnership (SSP) - } \\
\text { investigar aspectos da política e da } \\
\text { prática das parcerias, a partir do viés da } \\
\text { política baseada em evidências. }\end{array}$ & $\begin{array}{l}\text { School Sport Partnership } \\
\quad(\text { SSP), Inglaterra }\end{array}$ \\
\hline Understanding the impact of sport coaching on legacy. & $\begin{array}{l}\text { DUFFY, P.; } \\
\text { NORTH, J.; } \\
\text { MUIR, B. }\end{array}$ & 2013 & $\begin{array}{c}\text { International } \\
\text { Journal of Sport } \\
\text { Policy and Politics }\end{array}$ & $\begin{array}{l}\text { Enfatizar o UK Coaching Framework } \\
\text { como uma intervenção complexa e } \\
\text { analisar a avaliação de seu impacto nos } \\
\text { objetivos da política e legado. }\end{array}$ & $\begin{array}{l}\text { UK Coaching Framework, } \\
\text { Inglaterra }\end{array}$ \\
\hline $\begin{array}{l}\text { In pursuit of evidence-based policy and practice: a } \\
\text { realist synthesis-inspired examination of youth sport } \\
\text { and physical activity initiatives in England (2002- } \\
\text { 2010). }\end{array}$ & $\begin{array}{l}\text { LINDSEY, I.; } \\
\text { BACON, D. }\end{array}$ & 2016 & $\begin{array}{c}\text { International } \\
\text { Journal of Sport } \\
\text { Policy and Politics }\end{array}$ & $\begin{array}{l}\text { Sintetizar relatórios do governo para } \\
\text { entender melhor a implementação das } \\
\text { políticas esportivas para os jovens - } \\
\text { realist synthesis. }\end{array}$ & Inglaterra \\
\hline $\begin{array}{l}\text { Sport and Social Inclusion: Evidence-Based Policy and } \\
\text { Practice }\end{array}$ & COALTER, F. & 2017 & Social Inclusion & $\begin{array}{l}\text { Refletir sobre a experiência do autor no } \\
\text { Value of Sport Monitor, entre } 2004 \text { e } \\
2012 \text { - uma base de dados online, sobre } \\
\text { as políticas esportivas de inclusão social. }\end{array}$ & $\begin{array}{l}\text { Value of Sport Monitor, } \\
\text { Inglaterra }\end{array}$ \\
\hline $\begin{array}{l}\text { Sport policy evaluation: what do we know and how } \\
\text { might we move forward? }\end{array}$ & CHEN, S. & 2018 & $\begin{array}{c}\text { International } \\
\text { Journal of Sport } \\
\text { Policy and Politics }\end{array}$ & $\begin{array}{l}\text { Promover uma revisão crítica das bases } \\
\text { teóricas para algumas das abordagens } \\
\text { mais utilizadas na avaliação da política } \\
\text { de esporte. }\end{array}$ & - \\
\hline
\end{tabular}

Fonte: Autores. 
Research, Society and Development, v. 11, n. 1, e5111124491, 2022

(CC BY 4.0) | ISSN 2525-3409 | DOI: http://dx.doi.org/10.33448/rsd-v11i1.24491

No Quadro 2, é possível verificar os seis artigos selecionados, de acordo com os critérios de inclusão estabelecidos, com relação ao termo "process evaluation sports policy".

Quadro 2 - Artigos referentes ao termo de busca "process evaluation sports policy".

\begin{tabular}{|c|c|c|c|c|c|}
\hline \multicolumn{6}{|c|}{ BASE DE DADOS SCIVERSE SCOPUS DA ELSEVIER - Process Evaluation Sports Policy } \\
\hline ARTIGO & AUTOR(ES) & ANO & PERIÓDICO & OBJETIVO & PROGRAMA/PAÍS \\
\hline $\begin{array}{l}\text { Interventions implemented through sporting } \\
\text { organisations for increasing participation in sport. }\end{array}$ & $\begin{array}{l}\text { PRIEST, N.; } \\
\text { ARMSTRONG, R.; } \\
\text { DOYLE, J.; } \\
\text { WATERS, E. }\end{array}$ & 2008 & $\begin{array}{l}\text { Cochrane } \\
\text { Database of } \\
\text { Systematic } \\
\text { Reviews }\end{array}$ & 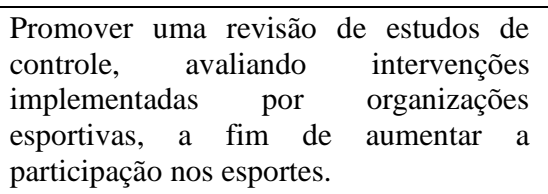 & - \\
\hline $\begin{array}{l}\text { Effectiveness of National Elite Sport Policies: A } \\
\text { Multidimensional Approach Applied to the Case } \\
\text { of Flanders. }\end{array}$ & $\begin{array}{l}\text { DE BOSSCHER, V.; } \\
\text { SHILBURY, D.; } \\
\text { THEEBOOM, M.; } \\
\text { VAN HOECKE, J.; } \\
\text { DE KNOP, P. }\end{array}$ & 2011 & $\begin{array}{l}\text { European Sport } \\
\text { Management } \\
\text { Quarterly }\end{array}$ & $\begin{array}{l}\text { Avaliar a efetividade das políticas de } \\
\text { esporte de elite, para além de seus } \\
\text { objetivos iniciais, utilizando o método } \\
\text { SPLISS. }\end{array}$ & Bélgica \\
\hline $\begin{array}{l}\text { Accounting for legacy: monitoring and evaluation } \\
\text { in sport in development relationships. }\end{array}$ & KAY, T. & 2012 & Sport in Society & $\begin{array}{l}\text { Examinar como o monitoramento e a } \\
\text { avaliação podem afetar materialmente o } \\
\text { legado olímpico, pensando o } \\
\text { desenvolvimento por meio do esporte. }\end{array}$ & Inglaterra \\
\hline $\begin{array}{l}\text { Ready, Steady, Go! Program, Italy: A Program } \\
\text { Impact Pathways (PIP) analysis. }\end{array}$ & $\begin{array}{l}\text { VERACINI, G.; } \\
\text { LEONARDI, E.; } \\
\text { GIROTTI, R. G.; } \\
\text { THRASHER, E. W. }\end{array}$ & 2014 & $\begin{array}{l}\text { Food and Nutrition } \\
\text { Bulletin }\end{array}$ & $\begin{array}{l}\text { Avaliar o programa Ready, Steady, Go!, } \\
\text { a partir de indicadores de impacto e dos } \\
\text { pontos de controle crítico de qualidade } \\
\text { (CCP). }\end{array}$ & Ready, Steady, Go! - Itália \\
\hline $\begin{array}{c}\text { The Health and Sport Engagement (HASE) } \\
\text { intervention and evaluation project: Protocol for } \\
\text { the design, outcome, process and economic } \\
\text { evaluation of a complex community sport } \\
\text { intervention to increase levels of physical } \\
\text { activity. }\end{array}$ & $\begin{array}{l}\text { MANSFIELD, L.; } \\
\text { ANOKYE, N.; } \\
\text { FOX-RUSHBY, J.; } \\
\text { KAY, T. }\end{array}$ & 2015 & BMJ Open & $\begin{array}{l}\text { Apresentar um protocolo para avaliação } \\
\text { do design, resultado, processo e viés } \\
\text { econômico do programa HASE, a fim de } \\
\text { aumentar o nível de atividade física em } \\
\text { determinada comunidade. }\end{array}$ & HASE, Inglaterra \\
\hline
\end{tabular}


Research, Society and Development, v. 11, n. 1, e5111124491, 2022

(CC BY 4.0) | ISSN 2525-3409 | DOI: http://dx.doi.org/10.33448/rsd-v11i1.24491

\begin{tabular}{|c|c|c|c|c|c|}
\hline $\begin{array}{l}\text { Information systems in local governments and } \\
\text { evaluation of public sport policies }\end{array}$ & $\begin{array}{l}\text { GARCÍA-FERNÁNDEZ, } \\
\text { R.; } \\
\text { GONZÁLEZ-DÍAZ, B.; } \\
\text { GONZÁLEZ-SALAS, J.; } \\
\text { PABLOS-RODRÍGUEZ, J. } \\
\text { L. } \\
\end{array}$ & 2015 & $\begin{array}{l}\text { Gestion y Politica } \\
\text { Publica }\end{array}$ & $\begin{array}{l}\text { Descrever o processo de implementação } \\
\text { de um sistema de contabilidade analítica } \\
\text { no Conselho Municipal de Esportes, da } \\
\text { prefeitura de Gijón, que pode ser um } \\
\text { ponto de partida para a avaliação de } \\
\text { políticas esportivas locais. }\end{array}$ & Espanha \\
\hline $\begin{array}{l}\text { An evaluation of equity and equality in physical } \\
\text { activity policies in four European countries. }\end{array}$ & $\begin{array}{l}\text { HÄMÄLÄINEN, R-M.; } \\
\text { SANDU, P.; } \\
\text { SYED, A. M.; } \\
\text { JAKOBSEN, M. W. }\end{array}$ & 2016 & $\begin{array}{l}\text { International } \\
\text { Journal for Equity } \\
\text { in Health }\end{array}$ & $\begin{array}{l}\text { Descrever como a equidade é } \\
\text { direcionada nas políticas de atividade } \\
\text { física em quatro países europeus. }\end{array}$ & $\begin{array}{c}\text { REPOPA - Dinamarca, } \\
\text { Finlândia, Romênia e } \\
\text { Inglaterra }\end{array}$ \\
\hline $\begin{array}{l}\text { A multidimensional approach to evaluate the } \\
\text { policy effectiveness of elite sport schools in } \\
\text { Flanders. }\end{array}$ & $\begin{array}{l}\text { DE BOSSCHER, } \mathrm{V} . ; \\
\text { DE KNOP, P; } \\
\text { VERTONGHEN, J. }\end{array}$ & 2016 & Sport in Society & $\begin{array}{l}\text { Avaliar a efetividade do Elite Sport } \\
\text { Schools em Flandres, a partir de uma } \\
\text { perspectiva da política multidimensional, } \\
\text { percebida pelos atletas. }\end{array}$ & Elite Sport Schools, Bélgica \\
\hline $\begin{array}{l}\text { A partnership-based evaluation of a community- } \\
\text { based youth sport and physical activity } \\
\text { programme. }\end{array}$ & $\begin{array}{l}\text { PARENT, M. M.; } \\
\text { HARVEY, J. }\end{array}$ & 2017 & Sport in Society & $\begin{array}{l}\text { Avaliar o componente de parceria do } \\
\text { esporte juvenil de base comunitária para } \\
\text { o programa de desenvolvimento, Kids in } \\
\text { Shape, através da aplicação do modelo } \\
\text { de gestão de parcerias. }\end{array}$ & Kids in Shape, Canadá \\
\hline
\end{tabular}

Fonte: Autores. 
Research, Society and Development, v. 11, n. 1, e5111124491, 2022

(CC BY 4.0) | ISSN 2525-3409 | DOI: http://dx.doi.org/10.33448/rsd-v11i1.24491

A partir da leitura dos textos selecionados, verificou-se que apenas dois deles - "Effectiveness of National Elite Sport Policies: A Multidimensional Approach Applied to the Case of Flanders" (De Bossher et. al., 2011) e “A multidimensional approach to evaluate the policy effectiveness of elite sport schools in Flanders" (De Bossher et. al., 2016) - se dedicaram especificamente ao esporte de alto rendimento. Nesse sentido, o resultado da busca por artigos, a partir de termos em Inglês, delimitou-se a dois textos, sendo ambos relacionados à metodologia do SPLISS (Sport Policy Factors Leading to International Sporting Success).

No que tange à busca de termos em Português, esta não foi muito bem-sucedida, na plataforma Scopus: o termo que obteve resultados foi "avaliação esporte”, com 14 resultados, porém, todos relacionados à performance física. Já os termos "avaliação processo política esporte", "avaliação processo esporte” e "evidência avaliação esporte” não apresentaram nenhum resultado. Tal dado levou à necessidade de busca diretamente nos sites das revistas científicas ${ }^{1}$ brasileiras da área de Sociologia do esporte, que contemplam grande parte das publicações que tangenciam a área de políticas públicas. Quais sejam, Revista Movimento (A2); Revista Brasileira de Educação Física e Esporte (B1); Revista Brasileira de Ciências do Esporte (B1); Revista Pensar a Prática (B2); Journal of Physical Education, da Universidade Estadual de Maringá (B2); e a Revista Motrivivência (B4). Os resultados podem ser vistos no Quadro 3, abaixo:

Quadro 3 - Resultados das buscas em revistas científicas brasileiras.

\begin{tabular}{|c|c|c|}
\hline PERIÓDICOS & $\begin{array}{c}\text { RESULTADO BRUTO POR TERMO DE } \\
\text { BUSCA }\end{array}$ & $\begin{array}{c}\text { ARTIGOS SELECIONADOS POR TERMO } \\
\text { DE BUSCA }\end{array}$ \\
\hline Revista Movimento & $\begin{array}{l}\text { Avaliação política }=14 \\
\text { Avaliação de processo }=22 \\
\text { Avaliação baseada em evidência }=0\end{array}$ & $\begin{array}{l}\text { Avaliação política }=1 \\
\text { Avaliação de processo }=1^{*} \\
\text { Avaliação baseada em evidência }=0\end{array}$ \\
\hline $\begin{array}{c}\text { Revista Brasileira de } \\
\text { Educação Física e Esporte }\end{array}$ & $\begin{array}{l}\text { Avaliação política }=97 \\
\text { Avaliação de processo }=315 \\
\text { Avaliação baseada em evidência }=15\end{array}$ & $\begin{array}{l}\text { Avaliação política }=0 \\
\text { Avaliação de processo }=0 \\
\text { Avaliação baseada em evidência }=0\end{array}$ \\
\hline $\begin{array}{l}\text { Revista Brasileira de } \\
\text { Ciências do Esporte }\end{array}$ & $\begin{array}{l}\text { Avaliação política }=24 \\
\text { Avaliação de processo }=110 \\
\text { Avaliação baseada em evidência }=58\end{array}$ & $\begin{array}{l}\text { Avaliação política }=0 \\
\text { Avaliação de processo }=0 \\
\text { Avaliação baseada em evidência }=0\end{array}$ \\
\hline Revista Pensar a Prática & $\begin{array}{l}\text { Avaliação política }=3 \\
\text { Avaliação de processo }=97 \\
\text { Avaliação baseada em evidência }=48\end{array}$ & $\begin{array}{l}\text { Avaliação política }=0 \\
\text { Avaliação de processo }=0 \\
\text { Avaliação baseada em evidência }=0\end{array}$ \\
\hline $\begin{array}{l}\text { Journal of Physical } \\
\text { Education }\end{array}$ & $\begin{array}{l}\text { Avaliação política }=0 \\
\text { Avaliação de processo }=28 \\
\text { Avaliação baseada em evidência }=5\end{array}$ & $\begin{array}{l}\text { Avaliação política }=0 \\
\text { Avaliação de processo }=1 \\
\text { Avaliação baseada em evidência }=0\end{array}$ \\
\hline Revista Motrivivência & $\begin{array}{l}\text { Avaliação política }=0 \\
\text { Avaliação de processo }=13 \\
\text { Avaliação baseada em evidência }=4\end{array}$ & $\begin{array}{l}\text { Avaliação política }=0 \\
\text { Avaliação de processo }=0 \\
\text { Avaliação baseada em evidência }=1\end{array}$ \\
\hline \multicolumn{2}{|c|}{ TOTAL DE ARTIGOS SELECIONADOS } & 3 \\
\hline
\end{tabular}

Fonte: Autores.

Os artigos selecionados, primariamente, foram: "Projetos de inclusão social através do esporte: notas sobre a avaliação" (Vianna \& Lovisolo, 2009), na Revista Movimento; “O programa Segundo Tempo em municípios brasileiros: indicadores de resultado no macrossistema" (Reverdito et. al., 2016), no Journal of Physical Education, da UEM; e "Projeto Cidades da Copa: movimento pelo legado esportivo dos megaeventos esportivos" (Paiva \& Rosseto Jr., 2013), na Revista Motrivivência.

\footnotetext{
${ }^{1}$ Considerou-se, também como critério de seleção das revistas, aquelas que possuem extrato.

* Mesmo artigo que apareceu na busca por "avaliação política", por este motivo, a soma total de artigos selecionados foi três e não quatro.
} 
Research, Society and Development, v. 11, n. 1, e5111124491, 2022

(CC BY 4.0) | ISSN 2525-3409 | DOI: http://dx.doi.org/10.33448/rsd-v11i1.24491

A julgar pela análise dos resumos dos artigos acima elencados, evidencia-se que, embora todos se refiram à avaliação de cunho acadêmico de projetos esportivos, estes estão no campo da inclusão social e participação, sem, portanto, dirigirem-se ao alto rendimento em específico.

O mesmo aconteceu com relação à busca realizada na plataforma Google Acadêmico. O texto selecionado foi um manual - "Sistema de monitoramento \& avaliação dos programas Esporte e Lazer da Cidade e Segundo Tempo do Ministério do Esporte" (Sousa, 2010) -, referente à avaliação de projetos também relacionados ao esporte de participação. Dessa forma, diante da busca realizada, com base nos termos em Português, nenhum texto se referia à avaliação direcionada ao esporte de alto rendimento.

\section{Conclusão}

Nesse sentido, é possível inferir uma escassa produção acerca da avaliação das políticas esportivas, em um sentido geral, e mais ainda em se tratando do esporte de alto rendimento. Entre as poucas abordagens que tratam deste assunto, as duas publicações referentes à análise com base no método SPLISS (De Bossher et. al., 2011, 2016) suscitaram a necessidade de realizar uma nova busca pela produção acadêmica. Embora a nova busca ainda enfatizasse a avaliação no esporte de alto nível, desta feita, foi utilizado como termo de busca a sigla "SPLISS". O resultado obtido foi de sete artigos, que buscavam analisar o sucesso esportivo em diferentes modalidades e países. Todavia, todos (Truyens et. al, 2014; Brouwers et al., 2015; Haro, Tanni, 2015; De Bossher, 2016; Storm et. al, 2016; De Bossher et. al, 2018; Nová, 2017) têm em comum o uso da metodologia SPLISS.

Tendo em vista que o SPLISS figura com centralidade entre as produções que buscam avaliar o esporte de alto rendimento, buscou-se, em um primeiro momento, pensar os fatores críticos de sucesso de pilares específicos, a fim de pensar um possível modelo para avaliação da política pública brasileira - mais especificamente, neste momento, o Programa BolsaAtleta. Entretanto, ao analisar tal metodologia com mais profundidade, percebeu-se que esta se refere a pensar a relação entre as políticas e o sucesso esportivo internacional; bem como buscar insights para os fatores que moldam o esporte de elite e os caminhos para o sucesso nas diferentes nações. Ou seja, o uso do SPLISS se direciona a pensar os sistemas esportivos de alto rendimento; ao passo que o objetivo da presente pesquisa é refletir sobre a avaliação de um programa específico do governo brasileiro.

Nesse sentido, em diálogo com os dados apresentados, destaca-se a necessidade de pesquisas futuras, a fim de refletir sobre as possibilidades e modelos de avaliação nas políticas públicas esportivas, haja vista a carência da área.

\section{Referências}

Brouwers, J., Sotiriadou, P, \& De Bosscher, V. (2015). Sport-specific policies and factors that influence international success: The case of tennis. Sport Management Review, 18 (3), 343-358.

Cavill, N., Foster, C., Oja, P., \& Martin, B. W. (2006). An evidence-based approach to physical activity promotion and policy development in Europe: contrasting case studies. IUHPE - Promotion \& Education, 13(2), 104-111.

Chen, S. (2018). Sport policy evaluation: what do we know and how might we move forward?.International Journal of Sport Policy and Politics, 10(4), 741759. DOI: $10.1080 / 19406940.2018 .1488759$.

Coalter, F. (2017). Sport and Social Inclusion: Evidence-Based Policy and Practice. Social Inclusion, 5(2), $141-149$.

Bosscher, V., Shilbury, D., Theeboom, M., Van Hoecke, J., \& De Knop, P. (2011). Effectiveness of National Elite Sport Policies: A Multidimensional Approach Applied to the Case of Flanders. European Sport Management Quarterly, 11(2), 115-141.

Bosscher, V., De Knop, P., \& Vertonghen, J. (2016). A multidimensional approach to evaluate the policy effectiveness of elite sport schools in Flanders. Sport in Society, 19 (10), 1596-1621. 
Research, Society and Development, v. 11, n. 1, e5111124491, 2022

(CC BY 4.0) | ISSN 2525-3409 | DOI: http://dx.doi.org/10.33448/rsd-v11i1.24491

Bosscher, V. (2016). A mixed methods approach to compare elite sport policies of nations. A critical reflection on the use of composite indicators in the SPLISS study. Sport in Society, 21 (2), 331-355. DOI: https://doi.org/10.1080/17430437.2016.1179729.

Bosscher, V., Shibli, S., \& Weber, A. Ch. W. (2018). Is prioritisation of funding in elite sport effective? An analysis of the investment strategies in 16 countries. European Sport Management Quarterly, 19 (2), 221-243. DOI: 10.1080/16184742.2018.1505926.

Duffy, P., North, J., \& Muir, B. (2013). Understanding the impact of sport coaching on legacy. International Journal of Sport Policy and Politics, 5(2), 165182.

García-Fernández, R., González-Díaz, B., González-Salas, J., \& Pablos-Rodríguez, J. L. (2015). Information systems in local governments and evaluation of public sport policies. Gestion y Politica Publica, Volume temático 2015, 171-204.

Gomes, I. S., \& Caminha, I. de O. (2014). Guia para estudos de revisão sistemática: uma opção metodológica para as Ciências do Movimento Humano. Movimento, 20(1), 395-411.

Hämäläinen, R-M., Sandu, P., Syed, A. M., \& Jakobsen, M. W. (2016). An evaluation of equity and equality in physical activity policies in four European countries. International Journal for Equity in Health, 15 (1), 1-13.

Kay, T. (2012). Accounting for legacy: monitoring and evaluation in sport in development relationships. Sport in Society, 15(6), 888-904.

Lindsey, I., \& Bacon, D. (2016). In pursuit of evidence-based policy and practice: a realist synthesis-inspired examination of youth sport and physical activity initiatives in England (2002-2010). International Journal of Sport Policy and Politics, 8(1), 67-90.

Liaton, K., Gregg, R., \& Lowther, J. (2013). Elite sports policy and coaching at the coalface. International Journal of Sport Policy and Politics, 5:3, $341-362$. DOI: $10.1080 / 19406940.2012 .735689$.

Mansfield, L., Anokye, N., Fox-Rushby, J., \& Kay, T. (2015). The Health and Sport Engagement (HASE) intervention and evaluation project: Protocol for the design, outcome, process and economic evaluation of a complex community sport intervention to increase levels of physical activity. BMJ Open, 5(10), 1-9.

Nová, J. Utilization of Research for Elite Sport in the Czech Republic. Sport Mont, 15 (3), 35-38. DOI: 10.26773/smj.2017.10.006.

Paiva, R. P., \& Rosseto Jr., A. J. (2013). Projeto Cidades da Copa: movimento pelo legado esportivo dos megaeventos esportivos. Motrivivência, 25(41), 7184

Parent, M. M., \& Harvey, J. (2017). A partnership-based evaluation of a community-based youth sport and physical activity programme. Sport in Society. 20(1), 7-29.

Priest, N., Armstrong, R., Doyle, J., \& Waters, E. (2008). Interventions implemented through sporting organisations for increasing participation in sport. Cochrane Database of Systematic Reviews, 16(3), 1-14.

Reverdito, R. S., Galatti, L. R., Lima, L. A. de, Nicolau, P. S., Scaglia, A. J., \& Paes, R. R. (2016). O Programa Segundo Tempo (PST) em municípios brasileiros: indicadores de resultado no macrossistema. Journal of Physical Education, 27(1), e-2754.

Smith, A., \& Leech, R. (2010). 'Evidence. What evidence?': evidencebased policy making and School Sport Partnerships in North West England. International Journal of Sport Policy and Politics, 2(3), 327-345.

Sousa, E. S. de, Noronha, V., Ribeiro, C. A., Teixeira, D. M. D., Fernandes, D. M., \& Venâncio, M. A. D. (2010). Sistema de monitoramento \& avaliação dos programas Esporte e Lazer da Cidade e Segundo Tempo do Ministério do Esporte. Belo Horizonte: O Lutador.

Storm, R. K., Nielsen, K., \& Thomsen, F. (2016). Can a small nation be competitive in the global sporting arms race? The case of Denmark, Managing Sport and Leisure, 21(4), 181-202. DOI: 10.1080/23750472.2016.1243993.

Taro, K. T., \& Hanni, E. (2015). Using a Comparative Method in Performance Audit for Evaluating Effectiveness of the Elite-Sports Policy: The Case of Estonia. Administrative Culture, 16 (1), 24-46.

Truyens, J., De Bossher, V., Heyndels, B., \& Westerbeek, H. (2014). A resource-based perspective on countries' competitive advantage in elite athletics. International Journal of Sport Policy and Politics, 6(3), 459-489, DOI: 10.1080/19406940.2013.839954.

Veracini, G., Leonardi, E., Girotti, R. G., \& Thrasher, E. W. (2014). Ready, Steady, Go! Program, Italy: A Program Impact Pathways (PIP) analysis. Food and Nutrition Bulletin, 35(3), S133-S138.

Vianna, J. A., \& Lovisolo, H. R. (2009). Projetos de inclusão social através do esporte: notas sobre a avaliação. Movimento, 15(3), 145-162. 